Research Article

\title{
Optimal Scheduling of a Microgrid Using Multiobjective Biogeography-Based Optimization Model and Algorithm with Adaptive Migration
}

\author{
Bo Li $(\mathbb{D}$, Jue Wang $(\mathbb{D}$, and Nan Xia \\ College of Information Science and Engineering, Dalian Polytechnic University, Dalian, China \\ Correspondence should be addressed to Jue Wang; ragnarok1876@163.com
}

Received 3 July 2020; Revised 23 August 2020; Accepted 25 August 2020; Published 5 October 2020

Academic Editor: Arkadiusz Zak

Copyright (c) 2020 Bo Li et al. This is an open access article distributed under the Creative Commons Attribution License, which permits unrestricted use, distribution, and reproduction in any medium, provided the original work is properly cited.

\begin{abstract}
Aiming at the important research topic of optimal scheduling in the microgrid field, the general model for multiobjective dynamic optimal scheduling of a microgrid is established with the objective of minimizing economic and environmental costs. On this basis, the model is organically integrated with constraint handling technology, multiobjective optimization, and biogeographybased optimization algorithm, and then a constrained multiobjective evolutionary model suitable for biogeography-based optimization is further established. The corresponding constraint handling mechanism, the determination method of habitat suitability index, and migration strategy are improved, and the convergence performance and the distribution uniformity of Pareto frontier for multiobjective evolutionary algorithm are effectively enhanced. Applied to the optimal scheduling of typical microgrid systems, the effectiveness of the proposed model and method is verified.
\end{abstract}

\section{Introduction}

Due to the global energy crisis and environmental pollution, the renewable clean energy such as solar and wind power has developed rapidly [1]. However, uncertain factors such as the intermittence and randomness of wind and light power output and the load fluctuation threaten the safe and stable operation of the power system $[2,3]$. Meanwhile, it is difficult to meet the power supply requirements in isolated islands or other remote areas. Therefore, microgrid, the small power generation and distribution system combining distributed power supply, energy storage device, load, and protection device, can effectively solve the above problems [4-6]. How to properly manage the distributed generation of microgrid and maximize the economic and environmental benefits of microgrid has become an important research topic $[7,8]$.

On account of the diversity of energy structure, distributed power types, and control methods in microgrid, the energy management and optimal operation of microgrid are of high complexity. Many scholars are committed to the research of microgrid energy management system. In [9], a simulation-based optimization method is proposed for the design of energy management system in photovoltaic gridconnected microgrid with heterogeneous occupancy. In [10], a sustainable scheduling problem for household microgrids with unscheduled distributed energy and responsive load demand is studied and the energy management system optimization algorithm based on mixed-integer linear programming (MILP) is proposed. In [11], the plug-in electric vehicles (PEVs) are introduced into microgrid, and a two-stage energy management strategy is proposed for the contribution of PEVs in the demand response plan of commercial building microgrid. In [12], a distributed requirements management system (DMS) is established to enhance any rule-based DMS through feedback behavior. Meanwhile, considering the multiobjective attribute of microgrid itself, the traditional single-objective optimal scheduling is difficult to adapt to the complex microgrid environment. At present, most of the research on the optimal operation of microgrid system focuses on the optimization of the model and the improvement of scheduling 
strategy and evolutionary strategy. In terms of solving the optimal scheduling model, the operation optimization of microgrid system is a nonlinear optimization problem with multiobjective and multiconstraints.

At present, the common intelligent optimization algorithms, genetic algorithm (GA) [13], particle swarming algorithm (PSO) [14], bacterial foraging algorithm (BFA) [15], and so on, are favored by scholars for their good parallelism, robustness, and global optimization ability but have their own advantages and disadvantages. It is still one of the problems worth studying to explore the optimization algorithm which is more suitable for solving the problem of optimal operation of microgrid. In [16], a multiobjective optimization model for microgrid operation management is built from three aspects of operating cost, pollutant emission, and operational risk level, and a niche multiobjective particle swarm optimization algorithm is proposed to solve the problem. In [17], a multiobjective economic scheduling model of cogeneration microgrid system is proposed, which considers the active and reactive power output and heat generation income. However, the above method is mainly optimized for the single objective and does not consider multiple objectives at the same time. In [18], the concept of energy and environmental benefits is introduced to modify the optimal scheduling model of power system including wind farms, and an improved particle swarm optimization algorithm combining tabu search and fuzzy processing technology is proposed. In [19], an improved Pareto genetic multiobjective optimization algorithm with vector module fitness function is proposed, which combines the idea of nondominated sorting, elitist strategy, and improved niche technology. In [20], a multiobjective optimization model of microgrid considering both the minimum $\mathrm{O} \& \mathrm{M}$ cost and the lowest environmental pollution is established, the multiobjective problem is transformed into a single-objective problem by using the linear weighted sum method, and the influence of different weight coefficients on the optimization results is discussed. In [21], the mathematical model of the optimal operation of microgrid based on improved gravitational search algorithm is proposed, taking into account several operation indexes such as economic costs, environmental benefits, and network losses. In [22], the multiobjective optimization model is solved by using the intensity Pareto evolutionary algorithm with three indexes of active network loss, pollution gas emission, and system voltage stability as optimization objectives. In [23], the mathematical model of microgrid operation is established, and the problem of optimal operation of microgrid is solved using improved adaptive firefly algorithm.

However, the above method is mainly optimized for the single objective and does not consider multiple objectives at the same time. Numerous multiobjective intelligent optimization algorithms, such as nondominated sorting genetic algorithm (NSGA-II) [24], multiobjective particle swarm algorithm (MOPSO) [25-27], nondominated sorting differential evolution (NSDE) [28], and game theory algorithms [29], are, respectively, used to solve the multiobjective optimal scheduling of microgrid. Although these methods belong to multiobjective optimization, they still suffer from the problem of falling into the local optimum frontier, which is unable to take into account both convergence and uniformity of the distribution.

In this paper, a general model for multiobjective dynamic optimal scheduling of microgrid is established with the objective of minimizing economic and environmental costs on the premise of satisfying load demand and microsource constraints. On top of this, the model is organically integrated with constraint handling technology, multiobjective optimization, and biogeography-based optimization (BBO) [30] algorithm. Then a constrained multiobjective evolutionary model suitable for $\mathrm{BBO}$ is further established and the corresponding constraint handling mechanism, the determination method of habitat suitability index, and adaptive migration strategy are effectively improved. Taking the typical microgrid system including photovoltaic array, wind turbine, fuel cell, microgas turbine, diesel engine, and storage battery as an example, the validity and reliability of the model and the algorithm proposed are analyzed.

\section{Optimal Scheduling Model of the Microgrid System}

The microgrid system used in this paper, which includes renewable and uncontrollable clean units such as photovoltaic array (PV) and wind turbine (WT), controllable nonclean units with high efficiency and small pollution such as fuel cell (FC), microgas turbine (MT), and diesel generator set (DE), and energy storage battery, is in island operation, and the internal load of microgrid is powered by each distributed generation unit. The goal of microgrid optimal scheduling is to optimize the output of microgrid power generation units under various constraints, so as to achieve the best economy and environmental protection of the system.

\subsection{Model of Each Power Generation Unit}

2.1.1. Wind Power Generation Model. The specific formula of the output power of wind turbine is shown as follows:

$$
P_{\mathrm{WT}}=\left\{\begin{array}{l}
0, v \leq c_{\mathrm{ci}}, v>v_{\mathrm{co}} \\
P_{v}, v_{\mathrm{ci}}<v \leq v_{\mathrm{co}}
\end{array}\right.
$$

where $v$ is the actual wind speed; $v_{\mathrm{ci}}$ and $v_{\mathrm{co}}$ are the cut-in and the cut-out wind speed. When the actual wind speed is lower than $v_{\mathrm{ci}}$ or higher than $v_{\mathrm{co}}$, the turbine stops; $P_{v}$ is the rated output power of wind turbine within the normal wind speed.

2.1.2. Photovoltaic Generation Model. Considering the randomness and intermittence of light and ambient temperature, the output power is calculated as follows:

$$
P_{\mathrm{PV}}=P_{S} \frac{S_{A}}{S_{0}}\left(1+k\left(T_{c}-T_{r}\right)\right) .
$$


In the formula, $P_{\mathrm{PV}}$ is the output power of photovoltaic cell; $P_{s}$ is the maximum output power under standard conditions; $S_{A}$ is the ambient light intensity; $S_{o}$ is the light intensity under standard conditions; $k$ is power temperature coefficient; $T_{C}$ is the working temperature of battery board; $T_{r}$ is the reference temperature.

2.1.3. Microgas Turbine Fuel Cost Model. The fuel cost of microgas turbine (MT) is shown by

$$
F_{\mathrm{MT}}=C_{\text {fuel }} \frac{1}{L} \frac{P_{\mathrm{MT}}}{\eta_{\mathrm{MT}}},
$$

where $F_{\mathrm{MT}}$ is the fuel cost of microgas turbine; $C_{\text {fuel }}$ is the price of natural gas; $L$ is the low calorific value of natural gas; $P_{\mathrm{MT}}$ and $\eta_{\mathrm{MT}}$ are the output power of MT and efficiency.

2.1.4. Fuel Cell Model. The energy consumption characteristic of the fuel cell model is similar to that of MT, and the fuel consumption can be expressed as shown in

$$
F_{\mathrm{FC}}=C_{\text {fuel }} \frac{1}{L} \frac{P_{\mathrm{FC}}}{\eta_{\mathrm{FC}}},
$$

where $F_{\mathrm{FC}}$ is the fuel cost of fuel cell; $P_{\mathrm{FC}}$ and $\eta_{\mathrm{FC}}$ are the output power and efficiency of FC.

2.1.5. Diesel Generator Fuel Cost Model. The fuel cost of diesel generator is shown by

$$
F_{\mathrm{DE}}=\alpha+\beta P_{\mathrm{DE}}+\gamma P_{\mathrm{DE}}^{2},
$$

where $F_{\mathrm{DE}}$ is the fuel cost of diesel generator; $\alpha, \beta$, and $\gamma$ are the fuel cost coefficient; $P_{\mathrm{DE}}$ is the output power of DE.

2.1.6. Storage Battery Model. In the economic dispatching of microgrid, the storage battery plays a role of peak load shifting in the load of microgrid and ensures continuous power supply of the system. The state of charge of a battery (SOC) is the ratio of its remaining capacity to its rated capacity. SOC at time $t+1$ is determined by battery capacity and SOC at time $t$ as follows; SOC charging formula can be expressed as

$$
\mathrm{SOC}_{t+1}=(1-\sigma) \mathrm{SOC}_{t}+\frac{\left(P_{\mathrm{ES}, t} \Delta t \eta_{\mathrm{IN}}\right)}{C}
$$

where $\sigma$ is the self-discharge rate; $P_{\mathrm{ES}, t}$ is the charge-discharge power at time $t ; \eta_{\mathrm{IN}}$ is the charge efficiency; and $C$ is total battery capacity within unit time $\Delta t$.

SOC discharging formula can be expressed as

$$
\mathrm{SOC}_{t+1}=(1-\sigma) \mathrm{SOC}_{t}-\frac{P_{\mathrm{ES}, t} \Delta t}{\left(C \eta_{\mathrm{OUT}}\right)}
$$

where $\eta_{\text {OUT }}$ is the discharge efficiency.

2.2. Optimization Model of the System. The purpose of microgrid optimal scheduling is to make the operating cost minimum of system and the environmental benefit maximum, by optimizing the output of each power supply in the microgrid with satisfying the load requirements and the constraints on the output of each distributed power.

\subsubsection{Objective Function}

(1) Minimize the Operating Cost

The operating cost is an important index to measure the economic operation of microgrid, which mainly includes fuel cost, maintenance cost, and low-voltage distribution and online electricity cost. It is shown in the following formula:

$$
\min F_{1}=\sum_{t=1}^{T} \sum_{i=1}^{N}\left(C_{F, i, t}+C_{\mathrm{OM}, i, t}+n C_{G, t}\right),
$$

where $F_{1}$ is the operating cost of microgrid; $T$ is the number of scheduling periods in the scheduling period; $N$ is the number of distributed power generation units; $C_{F, j, t}$ and $C_{\mathrm{OM}, j, t}$ are the fuel cost and maintenance cost of the $i$-th power generation unit at time $t ; C_{G, t}$ is the cost of power interaction between microgrid and large grid at time $t ; n$ is the cost factor in the system, when running in island mode, $n=0$.

(2) Minimize the Pollutant Treatment Cost

With the implementation of the strategy of sustainable development, environmental benefits are increasingly valued. In this paper, the pollution degree is defined to measure the harm degree of $\mathrm{CO}_{2}, \mathrm{SO}_{2}, \mathrm{NO}_{x}$, and other pollution gases to the environment caused by the equivalent discharge from MT, DE, and absorbing power of low-voltage distribution grids. Since PV and WT, as new energy units, do not produce pollution gas during operation, the pollution degree is not considered. The object function formula is as follows:

$$
\min F_{2}=\sum_{t=1}^{T} \sum_{j=1}^{M} C_{j} \sum_{i=1}^{N} K_{i j} P_{i, t},
$$

where $F_{2}$ is the treatment cost of pollutant discharge; $M$ is the type of pollutants discharged such as $\mathrm{CO}_{2}, \mathrm{SO}_{2}$, and $\mathrm{NO}_{x} ; \mathrm{C}_{j}$ is the cost of discharged pollutants; $K_{i j}$ is the discharge coefficient of pollutant $j$ in the $i$-th power generation unit; and $P_{i, t}$ is the generation capacity of the $i$-th unit at time $t$.

\subsubsection{Constraints}

(1) The power balance constraint is as follows:

$$
\sum_{i=1}^{N} P_{i, t}+P_{\mathrm{ES}, t}-P_{L, t}=0,
$$

where $P_{i, t}$ is the power output of each micropower source at time $t ; P_{\mathrm{ES}, t}$ is the output power of energy storage battery; when $P_{\mathrm{ES}, t}>0$, it means discharging; $P_{L, t}$ is the microgrid load at time $t$. 
(2) The output power constraint of micropower supply is as follows:

$$
P_{\text {imin }} \leq P_{i} \leq P_{i \max }
$$

where $P_{i \min }$ and $P_{i \max }$ are the minimum and maximum output power, respectively.

(3) The climbing speed constraint is as follows.

When the load increases,

$$
P_{i, t}-P_{i, t-1} \leq R_{i u p}
$$

When the load is reduced,

$$
P_{i, t-1}-P_{i, t} \leq R_{i \text { down }}
$$

Here,d $R_{\text {iup }}$ and $R_{\text {idown }}$ are the upper and lower limits of climbing rate of the $i$-th power generation unit.

(4) The charging and discharging constraints of energy storage battery are as follows.

To ensure the battery life, charging and discharging must follow the following formula:

$$
\mathrm{SOC}_{i \min } \leq \mathrm{SOC}_{i, t} \leq \mathrm{SOC}_{i \max },
$$

where $\mathrm{SOC}_{i \min }$ and $\mathrm{SOC}_{i \max }$ are the minimum and maximum capacity of energy storage battery.

\section{Dynamic Optimal Scheduling of a Microgrid Based on Biogeography-Based Optimization}

3.1. Constrained Multiobjective Optimization Problem. A constrained multiobjective optimization problem (CMOP) with minimization can be defined as

$$
\begin{array}{ll}
\operatorname{minimize} & y=F(x)=\left(f_{1}(x), f_{2}(x), \ldots, f_{M}(x)\right)^{T}, \\
\text { subject to } & g_{j}(x) \leq 0, \quad j=1,2, \ldots, q, \\
& h_{j}(x)=0, \quad j=q+1,2, \ldots, m,
\end{array}
$$

where $x=\left(x_{1}, \ldots, x_{n}\right) \in X \subset R^{n}$ is $n$-dimensional vector of decision variables; $X$ is the decision space; $y=\left(y_{1}, y_{2}, \ldots, y_{M}\right) \in Y \subset R^{M}$ is $M$-dimensional objective vectors; $Y$ is the objective space; $F(x)$ defines the function which is mapped from the decision space to the objective space; $g(x)$ and $h(x)$ define $q$ inequality constraints and $m-$ $q$ equality constraints.

3.2. Modified Multiobjective Biogeography-Based Optimization Model and Algorithm. In view of the fact that the $\mathrm{BBO}$ algorithm itself does not have the ability to deal with multiobjective optimization and constrained multiobjective optimization problems, a multiobjective optimization model is proposed in this paper, which is suitable for the $\mathrm{BBO}$ algorithm based on the new multiobjective constraint handling mechanism. In addition, considering the greater effect of the performance of the evolutionary strategy on the overall performance of the algorithm, in order to improve the constrained optimization ability of multiobjective optimization, the efficiency of BBO algorithm must be ensured.

\subsubsection{Constrained Multiobjective Handling Mechanism.}

The main idea of $\varepsilon$ constraint handling technology is to measure the degree of the individual by comparing the level parameter $\varepsilon$ with the individual constraint violation degree, enlarge the constraint region effectively under the allowable constraint violation degree, and promote more excellent and infeasible individuals with lower constraint violation degree to participate in the evolution, so as to guide the whole group to approach the feasible region. For the individual $x=\left(x_{1}, \ldots, x_{n}\right)$ in the population $H=\left\{x_{1}, x_{2}, \ldots, x_{\mathrm{NP}}\right\}$, the degree of violation $\varphi_{j}(x)$ of the $j$ th constraint is shown in

$$
\varphi_{j}(x)=\left\{\begin{array}{l}
\max \left(0, g_{j}(x)\right), \quad j=1,2, \ldots, q, \\
\max \left(0,\left|h_{j}(x)\right|-\delta\right), \quad j=q+1, \ldots, m .
\end{array}\right.
$$

Then, the comprehensive constraint violation degree of the individual $\phi(x)$ is defined as the linear addition of each constraint violation degree.

However, the $\varepsilon$ constraint handling method based on linear addition and simple constraint violation has its drawback in solving CMOPs that ignore the difference of the degree of difficulty satisfying the different constraints. In order to reflect the real constraint violation degree more objectively, a new self-adaptive weighted $\varepsilon$ comprehensive constraint violation degree determination method is proposed as shown in

$$
\phi(x)=\sum_{j=1}^{m} \frac{n_{j_{-} \text {fea }}}{\mathrm{NP}} \times\left(\max \left(0, \varphi_{j}(x)-\varepsilon_{j}(t)\right)\right),
$$

where NP is the total number of individuals in the current population; $n_{j_{f} \text { fea }}$ is the number of individuals satisfying the $j$ th constraint $\left(\varphi_{j}(x)=0\right) ; \varepsilon_{j}(t)$ is the level parameter of the $j$ th constraint at $t$ time, as determined by formula (5) in the following chapter.

In order to further measure the quality of individuals, we also need to integrate the multiobjective Pareto dominance into the comparison among individuals according to the characteristics of CMOPs. Therefore, for the individual $x_{1}$ and $x_{2}$ in population $H$, the $\varepsilon$ constraint dominance relationship combining the Pareto dominance with the adaptive constraint violation degree $\phi(x)$ is shown in

$$
x_{1}>{ }_{\varepsilon} x_{2} \Leftrightarrow\left\{\begin{array}{l}
x_{1}>x_{2}, \phi\left(x_{1}\right)=\phi\left(x_{2}\right), \\
\phi\left(x_{1}\right)<\phi\left(x_{2}\right) .
\end{array}\right.
$$

In the new $\varepsilon$ constraint multiobjective handling mechanism proposed above, each constraint condition $j$ needs to be given a level parameter epsilon $\varepsilon_{j}$. In view of the above 
problems of the original level parameter $\varepsilon$ which is based on the nonlinear decrease of iterations, a new method to determine $\varepsilon$ is shown in

$$
\varepsilon_{j}(t)= \begin{cases}\frac{\sum_{i=1}^{N P}\left(\varphi_{j}^{t}(x) \times\left(\varphi_{j \max }^{t}-\varphi_{j}^{t}(x) / \varphi_{j \max }^{t}-\varphi_{j \min }^{t}\right)\right)}{\sum_{i=1}^{\mathrm{NP}}\left(\left(\varphi_{j \max }^{t}-\varphi_{j}^{t}(x) / \varphi_{j \max }^{t}-\varphi_{j \min }^{t}\right)\right)}, & \varphi_{j \max }^{t} \neq \varphi_{j \min }^{t}, \\ 0, & \varphi_{j \max }^{t}=\varphi_{j \min }^{t},\end{cases}
$$

where $\varphi_{j}^{t}(x)$ is the constraint violation of individual $X_{i}$ at time $t ; \varphi_{j \text { max }}^{t}$ and $\varphi_{j \text { min }}^{t}$ are the maximum and minimum of $\varphi_{j}^{t}(x)$. For the $j$ th constraint, all of $\varepsilon_{j}$ of each generation is adaptively adjusted for the changes of feasible region according to the $j$ th constraint violation degree of the individual $x \cdot \varphi_{j}(x)$ participates in regulating the level parameter in the form of its proportion in the violation degree of individuals. The larger $\varphi_{j}(x)$ may affect the level parameter $\varepsilon_{j}$ with a smaller proportion, and vice versa.

\subsubsection{Modified Determination Method of Habitat Suitability} Index. In the BBO algorithm, habitat suitability index (HSI) is an indicator of measuring the habitat quality and also an important factor affecting the distribution and migration of habitat populations. For CMOPs, in consideration of the characteristics of multiobjectives, it is necessary to determine HSI by combining Pareto dominance among habitat individuals. However, it is far from enough to define the HSI which is not enough to evaluate the quality of the habitat in this way and the constraints should also be taken into account fully. Thus, it is ideal to design a determination mechanism of HSI, which can take into account the Pareto domination relationship and the degree of constraint satisfaction of the individual itself.

For this purpose, this paper proposes a new determination method of habitat suitability index HSI on the basis of the idea of $\varepsilon$ constraint domination mentioned above. First of all, the individuals in habitat population $H=\left(x_{i}, i=1,2, \ldots, \mathrm{NP}\right)$ are ranked in ascending order in accordance with the adaptive $\varepsilon$ weighting constraint violation and form a new habitat population $H$. Then, the $\varepsilon$ feasible habitat population $H_{\text {efea }}=\left\{x_{i} \mid \phi\left(x_{i}\right)=0\right\}$ of the current new population $H$ is determined and the nondominance degree $F_{i}$ of individual $x_{i}$ in $H_{\text {efea }}$ is calculated by

$$
F_{i}=\sum_{x_{i} \in H_{\text {efea }}, x_{j}>x_{i}}\left(\frac{1}{\mathrm{NP}}\left|\left\{k \mid x_{j}, x_{k} \in H_{\varepsilon f e a}, \wedge x_{j}>x_{k}, H_{\varepsilon f e a}=\left\{x_{i} \mid \phi\left(x_{i}\right)=0\right\}\right\}\right|\right), \quad i, j, k \in\{1,2, \ldots, \mathrm{NP}\}
$$

where $x_{i}, x_{j}, x_{k} \in H$ is the habitat individual including $n$ dimensional suitability index variables (SIV). Finally, the HSI $G_{i}$ of the individual $x_{i}$ in the new $H$ is determined by

$$
\begin{aligned}
G_{i} & =\max \left(\phi\left(x_{i}\right), 0\right)+F_{i}^{\prime}, \\
F_{i}^{\prime} & =\left\{\begin{array}{l}
F_{i}, \phi\left(x_{i}\right)=0, \\
F_{\text {max }}, \phi\left(x_{i}\right) \neq 0 .
\end{array}\right.
\end{aligned}
$$

3.2.3. Determination Method of Migration Rate. The determination mechanism for the migration rate of the original $\mathrm{BBO}$ does not apply to CMOPs, which can only reflect the order of individuals but ignores the current evolutionary state. Thus, it is impossible to effectively evaluate the quality of individuals. When the gap between individuals is large or the distribution is unbalanced, it is very easy to make a wrong assessment of the quality of individuals, resulting in the fact that the information of better individuals cannot be retained or worse individuals participate in evolution too early.

In order to reflect the quality of individuals in real time and ensure that the information of the better individuals can be made full use of in different evolutionary stages, a method of determination of immigration rate $\lambda$ and the emigration rate $\mu$ is shown in the following formula:

$$
\left\{\begin{array}{l}
\lambda_{i}=\left(\frac{G_{i}-G_{\min }}{G_{\max }-G_{\min }}\right)^{\mathrm{NP}^{1-(\alpha+1)^{\eta}}} \\
\mu_{i}=1-\lambda_{i}
\end{array}\right.
$$

where $G_{i}$ is the HSI of $x_{i} ; \alpha$ is the nondominated rate of $H_{\varepsilon f e a}$, the proportion of nondominated $\varepsilon$ feasible individuals in the number $n_{\varepsilon f e a}$ of $H_{\varepsilon f e a} ; G_{\max }$ and $G_{\min }$ are the maximum and minimum HSI of $G_{i} ; \eta$ is the $\varepsilon$ feasible rate, the proportion of $n_{\varepsilon f e a}$ in NP. The HSI of normalized individuals is used to adjust the immigration rate in combination with the proportion of $\varepsilon$ feasible individuals and the proportion of $\varepsilon$ feasible nondominated individuals in the current population.

According to the difference of the evolutionary stages, the role of adaptive migration rate determination 
mechanism in the evolution of populations can be attributed to the following three scenarios:

(a) At the beginning of population evolution, when the number of $\varepsilon$ feasible individuals is small, the adaptive migration rate can give more opportunities to $\varepsilon$ feasible individuals or $\varepsilon$ infeasible individuals with smaller constraint violation to guide the evolution of the population according to the quality of individuals and rapidly evolve the individuals with larger constraint violation to approach the feasible region from different directions

(b) As the number of $\varepsilon$ feasible individuals gradually increases, the adaptive migration rate can guide the $\varepsilon$ feasible individuals to move towards the direction with better nondomination degree and guide the remaining $\varepsilon$ infeasible individuals to approach the feasible region or even enter the feasible region, so as to efficiently evolve the less nondominant individuals and the remaining individuals with $\varepsilon$ infeasible

(c) When the population is composed of feasible individuals and the number of nondominant feasible individuals gradually increases or even spreads over the population, the more concentrated nondominant feasible individuals have more opportunities to participate in evolution and thus become more evenly distributed

3.2.4. Modified Migration Strategy. For CMOPs, when the search space is nonconvex or disconnected, the algorithm often falls into a certain subregion and the solution found is a local Pareto optimal solution, which makes it very difficult for the algorithm to solve CMOPs and poses higher requirements for the evolutionary mechanism of the algorithm. In order to overcome the effects of the constraints, the immigration rate with $\varepsilon$ feasible and Pareto dominant information is used as a control parameter to adjust the current habitat individual $x_{i}$ and the emigrated habitat individual $x_{k}$ adaptively. In the meantime, the difference vector of exploiting the new solution is added and the disturbance of which is dynamically adjusted by the time factor. The modified dynamic migration strategy is shown in

$$
\begin{aligned}
x_{i j}= & \mu_{i} x_{k j}+\lambda_{i} x_{i j}+\left(r_{\min }+r(t)\left(r_{\max }-r_{\min }\right)\right) \\
& \times\left(x_{r 1 j}-x_{r 2 j}\right), \\
r(t)= & (1-\beta) \times r(t-1)+\beta,
\end{aligned}
$$

where $x_{i j}$ is the $j$ th-SIV of $x_{i}$; $r$ is the time factor that can control the amplitude of the difference vector perturbation; $r_{\max }$ and $r_{\min }$ are the maximum and the minimum of $r ; r_{1}$ and $r_{2}$ are the random number between 1 and NP; and $\beta$ is the specified lower normal number.

The proposed dynamic migration operator can dynamically adjust the individual's evolutionary trend at different evolutionary periods. At the beginning of evolution, the first half of formula (23) is used to rapidly guide the evolution of the population towards the better direction, and the second half is used to perturb the population by the small amount of difference vector. In this way, when the number of $\varepsilon$ feasible individuals is small, the population can fully absorb the information of the better $\varepsilon$ infeasible individuals or even $\varepsilon$ feasible individuals, which ensures the rapid population evolution towards the $\varepsilon$ feasible region. With the continuous evolution, as the number of $\varepsilon$ feasible individuals in the population increases and the presence of nondominant $\varepsilon$ feasible individuals gradually increases with evolution, the population can continue to converge towards the nondominant frontier of the feasible region at high speed.

However, when the population has evolved to a certain stage where the population is almost entirely composed of nondominant feasible individuals, in order to make the nondominant feasible individuals closer to the Pareto frontier and obtain a better distribution, at this time, the algorithm mainly uses the time factor to dynamically adjust the difference vector in the second half of formula (23) to realize the full exploitation of new individuals. The gradual increase in the magnitude of the deviation perturbation can accelerate the spread of individuals to sparse regions, improve the population diversity, and provide more opportunities for escaping from local extremes.

3.3. The Improved Algorithm Flow. According to the analysis of multiobjective optimization model above, the optimal scheduling of a microgrid is a complex nonlinear optimization problem with multiobjective and multiple constraints. The specific procedure of dynamic optimal scheduling of microgrid based on improved constraint multiobjective biogeography-based optimization model and algorithm with adaptive migration (MOBBO-AM) is shown in Figure 1.

Step 1. Set the relevant data and operating parameters of the microgrid, which involves the thermoelectric load, the power generation of wind turbines and photovoltaic cells, and so on. And set the relevant constraints of each micropower source.

Step 2. Initialize the relevant parameters of the proposed algorithm, which involves $m_{\max }, r_{\max }, r_{\min }$, and $\beta$. Randomly generate NP individuals as the initial population $H=\left(x_{i}, i=1,2, \ldots, N P\right)$. For each $H$, it is generated by a micropower in different periods and its dimensions are $N \times T . N$ is the number of distributed generation units to be optimized in the microgrid, and $T$ is the total scheduling period.

Step 3. Calculate the objective function $F\left(x_{i}\right)=\left(f_{1}\left(x_{i}\right), \ldots, f_{M}\left(x_{i}\right)\right)$ and the constraint violation degree $\gamma\left(x_{i}\right)=\left(\varphi_{1}\left(x_{i}\right), \ldots, \varphi_{m}\left(x_{i}\right)\right)$ of each habitat individual $x_{i}$.

Step 4. Calculate the $\varepsilon$ value under each constraint of $x_{i}$, and determine the $\varepsilon$ constraint violation degree $\phi\left(x_{i}\right)$ of $x_{i}$. Sort $x_{i}$ by its $\phi\left(x_{i}\right)$ in ascending order, and reform a new population $H$. According to $\phi\left(x_{i}\right)$, determine the feasible population $H_{\varepsilon f e a}$ in $H$, calculate the nondominated degree $F_{i}$ 


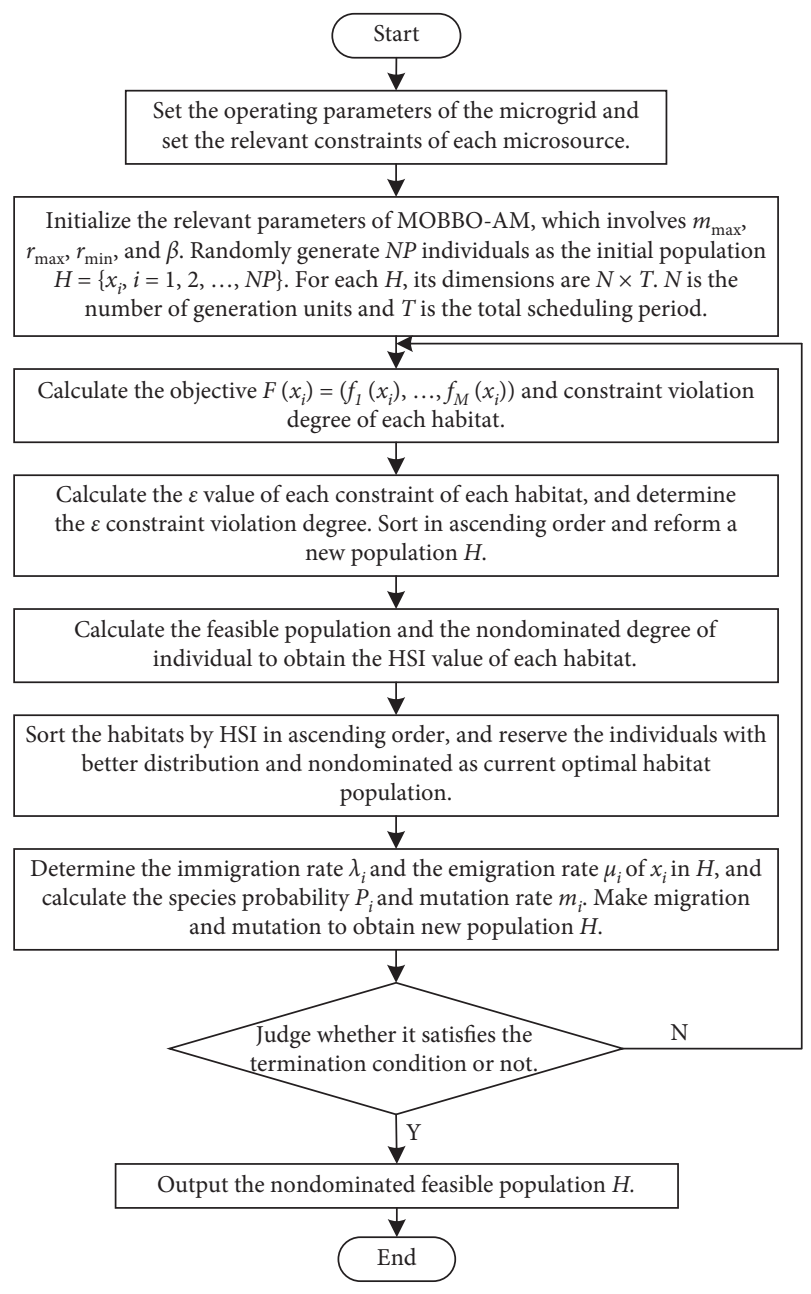

Figure 1: Results of the environmental optimal scheduling.

of individual in $H_{\varepsilon f e a}$, and thus obtain the HSI value $G_{i}$ of each habitat individual $x_{i}$.

Step 5. Sort the habitat individuals of $H$ by HSI in ascending order, and reserve the NP individuals with better distribution and nondominated as current optimal habitat population $H$.

Step 6. Determine the species immigration rate $\lambda_{i}$ and the emigration rate $\mu_{i}$ of $x_{i}$ in $H$, and calculate the species probability $P_{i}$ and mutation rate $m_{i}$. On the basis of immigration rate $\lambda_{i}$ and the emigration rate $\mu_{i}$, make migration and mutation, and thus obtain new population $H$. Judge whether it satisfies the termination condition or not. If it satisfies, then go to Step 7; otherwise, go to Step 3.

Step 7. Output the nondominated feasible population $H$.

\section{Calculation Example Analysis}

4.1. Basic Data of Calculation Example and Parameter Setting. In order to verify the effectiveness of the above-mentioned model, a typical microgrid system, including photovoltaic cells, wind turbines, fuel cells, microgas turbines, diesel generator sets, energy storage battery, and load, carried out the optimal scheduling.

In this paper, the output of each distributed generation (DG) unit in 24 periods of a day is taken as the optimization object, and its parameters are shown in Table 1. The typical daily load data is selected, and the prediction curves of load, temperature, wind speed, and light intensity are shown in Figure 2. Meanwhile, on the premise of maximizing the use of renewable energy, PV and WT work in maximum power point trace mode (MPPT), and the output curve of WT and PV is shown in Figure 3. The discharge data, pollutant value standard, and penalty level of pollutants are shown in [16]. The relation curves of the environmental treatment cost and the output power, the fuel cost, and the output power of controllable microsource are shown in Figures 4 and 5, respectively. The parameters of $\mathrm{MOBBO}-\mathrm{AM}$ algorithm proposed in this paper are set as follows: the initial population size $N P$ is set as $100, m_{\max }=0.005, r_{\max }=0.9$, $r_{\min }=0.4$, and $\beta=0.02$, and the maximum number of iterations is 500 .

From Figures 4 and 5, it can be seen that FC has the lowest environmental pollution treatment cost during operation, followed by MT, and DE has a higher environmental cost. For the operating cost, the fuel cost of MT and FC is higher in operation, in which MT is slightly higher than FC, while the fuel cost of DE is lower.

4.2. Simulation Results and Analysis. In order to realize the comprehensive optimal scheduling of economy and environmental protection of microgrid system, the above microoptimal scheduling model combined with improved algorithm is used for experiments, and the optimal Pareto frontier of operating cost and environment cost is shown in Figure 6.

As can be seen from Figure 6, the environmental cost range is about $\$ 40$ to $\$ 140$, and the operating cost range is about $\$ 270$ to $\$ 470$ on the Pareto frontier. When the operating cost is low, the environmental cost may rise, and vice versa. If the microgrid operates at the lowest operating cost, the total cost of simple summation is about $\$ 410$, while the total cost of operating microgrid at the lowest environmental cost is about $\$ 510$. Therefore, taking into account the environmental cost and operating cost, it is likely to obtain the optimal solution with lower comprehensive operation cost or equivalent comprehensive cost but less environmental cost by choosing the compromise solution on Pareto frontier. In this example, when the operating cost is $\$ 290$, the environmental cost is only $\$ 83$, for a total cost of $\$ 373$, which not only reduces the environmental impact but also maximizes the economy. It is not difficult to see from the figure that the relationship between operating cost and environmental cost is contradictory, and with the increase of operating cost of microgrid, the pollutant treatment cost of microgrid is relatively reduced. It is necessary to comprehensively consider various factors to choose a compromise between them. The algorithm in this paper can not only obtain the effective convergence solution set but also obtain the Pareto frontier evenly and widely distributed, which provides a more 
TABle 1: Parameters of generation units.

\begin{tabular}{|c|c|c|c|c|}
\hline \multicolumn{3}{|c|}{ Output power/kW } & \multirow{2}{*}{ Depreciable life/a } & \multirow{2}{*}{ Operation and maintenance coefficient $(\$ / \mathrm{kW} \cdot \mathrm{hr})$} \\
\hline DG & $P_{\max }$ & $P_{\min }$ & & \\
\hline MT & 130 & 15 & 10 & 0.0060 \\
\hline FC & 125 & 12 & 10 & 0.0042 \\
\hline BAT & 40 & -40 & 10 & 0.0064 \\
\hline PV & 40 & 0 & 20 & 0.0014 \\
\hline WT & 40 & 0 & 10 & 0.0042 \\
\hline DEG & 125 & 0 & 15 & 0.0113 \\
\hline
\end{tabular}

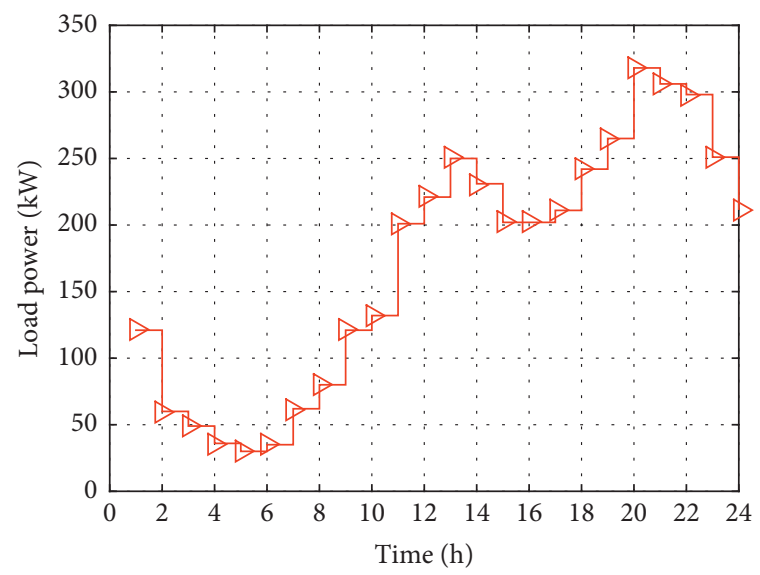

(a)

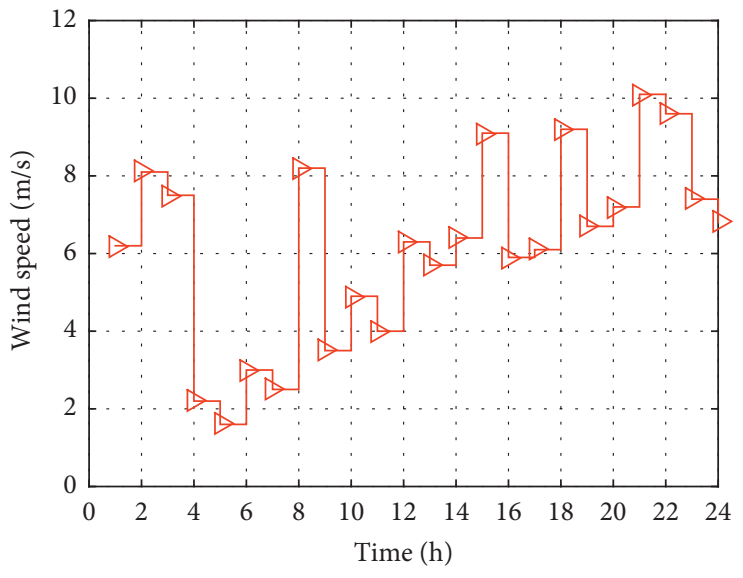

(c)

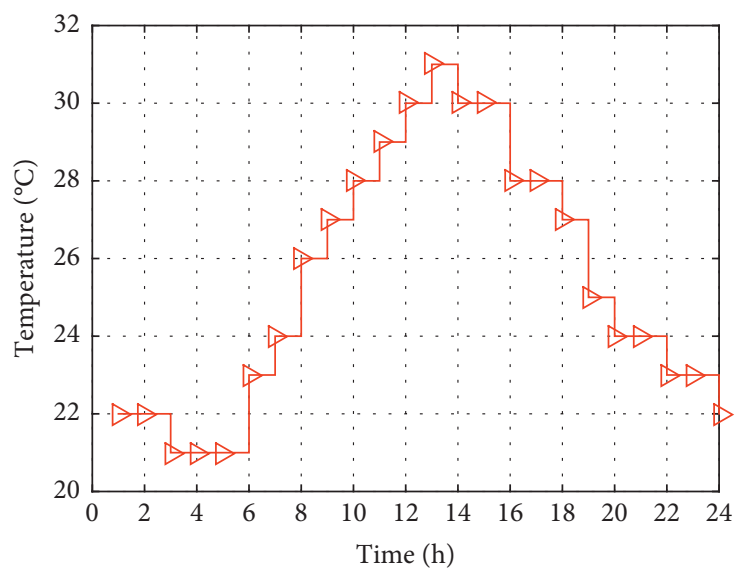

(b)

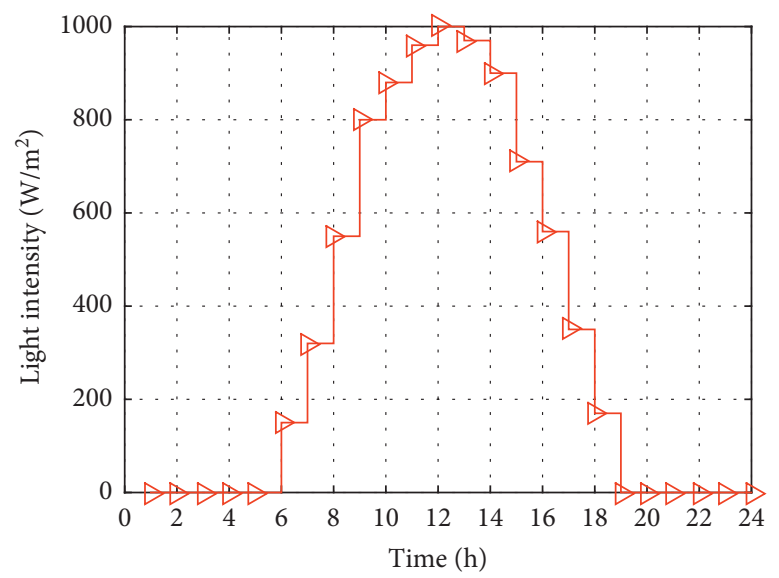

(d)

Figure 2: The prediction curves of load, temperature, wind speed, and light intensity. (a) $24 \mathrm{~h}$ load output curve, (b) $24 \mathrm{~h}$ temperature curve, (c) $24 \mathrm{~h}$ wind speed curve, and (d) $24 \mathrm{~h}$ light intensity curve.

comprehensive and reasonable choice for the economic and environmental operation and dispatching of microgrid.

Three representative solutions are chosen on the Pareto frontier in Figure 6 and their corresponding microgrid scheduling scenarios are analyzed briefly, where Figures 7 to 9 are, respectively, the cases with lower operating cost, lower environmental cost, and minimum comprehensive cost. Afterward, the economic scheduling with the minimum operating cost, the environmental scheduling with the minimum environmental cost, and the comprehensive optimal scheduling are analyzed, respectively, and the results are shown in Table 2.

It is not difficult to see from Figure 7 that, considering the economic cost and environmental protection cost, the operation of microgrid needs to be analyzed in different periods. At 1:00 to 7:00 of electricity valley time, there is no light but there is wind at this moment; PV does not generate electricity, but WT can supply power continuously. Considering the supply and demand of electricity, FC and MT keep the low power operation, DE replenishes the remaining 


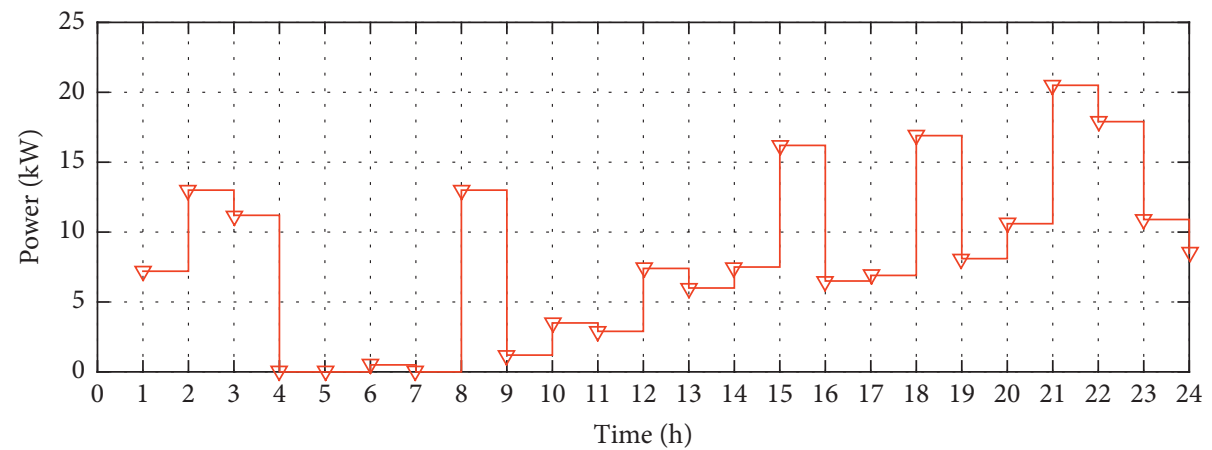

(a)

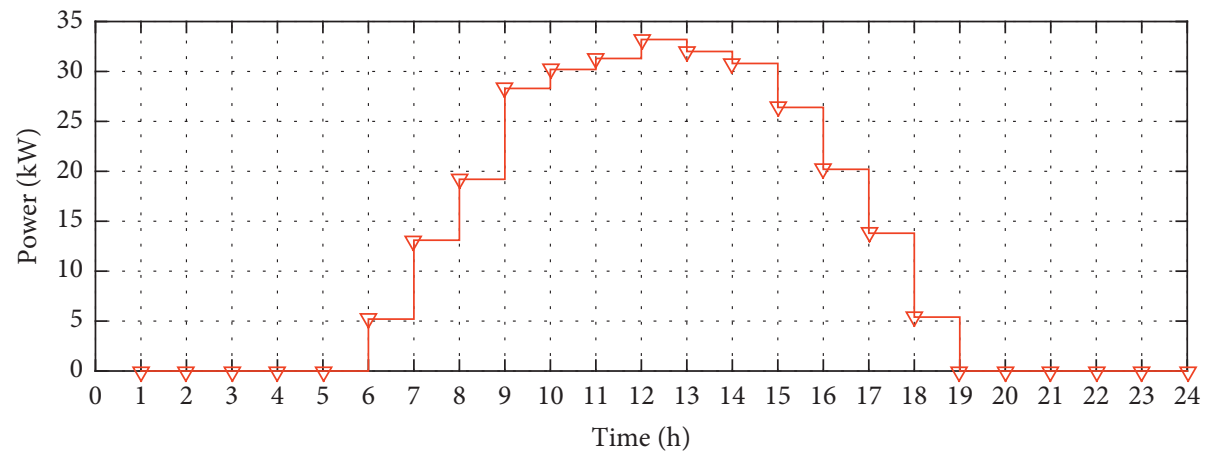

(b)

Figure 3: Prediction curves of WT and PV output. (a) $24 \mathrm{~h}$ WT output curve and (b) $24 \mathrm{~h}$ PV output curve.

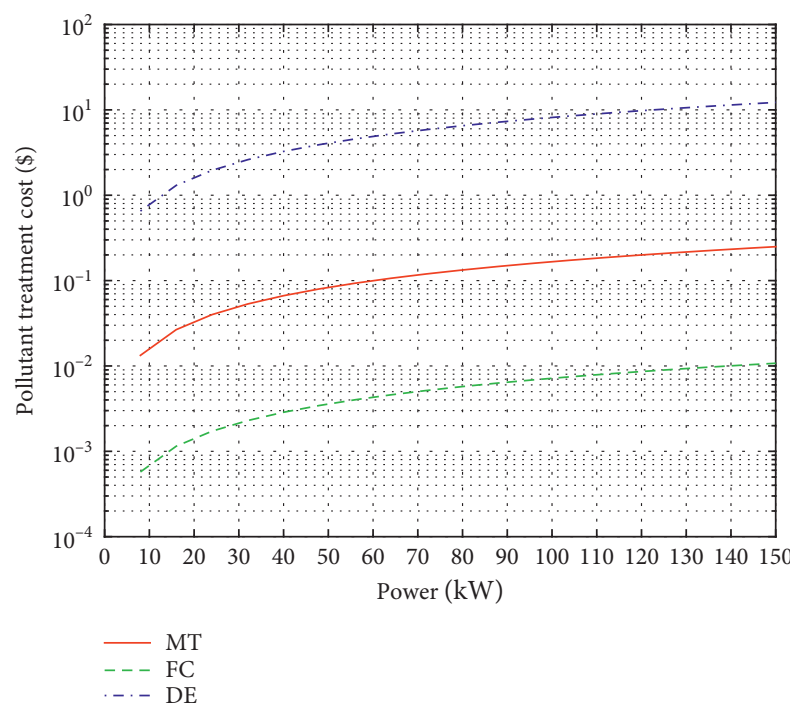

FIGURE 4: The relation curves of environmental treatment cost and the output power.

electricity demand, and the battery remains charged. After 8: 00 , electricity consumption continues to rise; although the light intensity gradually increases, PV and WT still cannot meet the demand for electricity. When DE power gradually increases, the battery starts to discharge, which is used to meet the load demand for electricity. After 11:00, when the power consumption continues to rise to the point where $\mathrm{DE}$

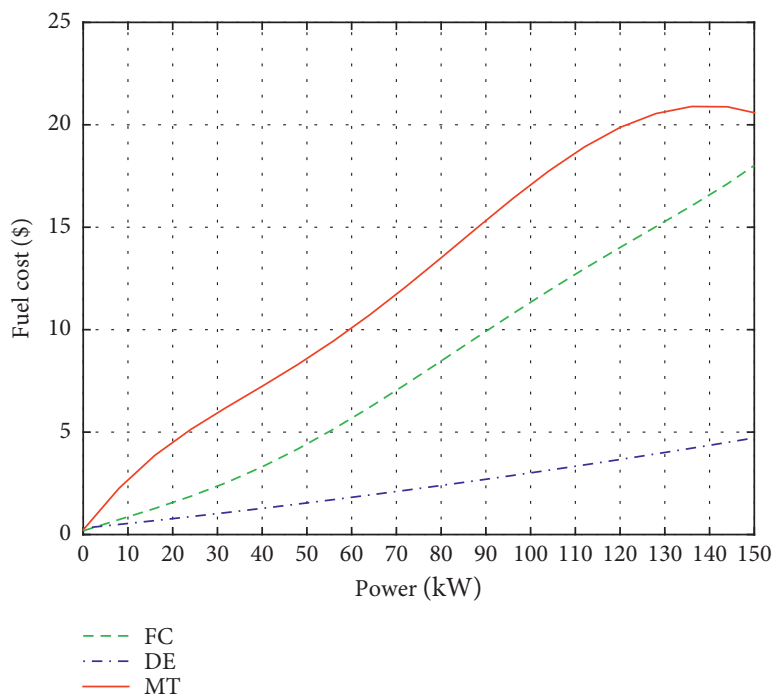

FIgURE 5: The relation curves of fuel cost and the output power.

is close to full load, FC begins to replenish the power demand while MT still operates at a lower power.

At 15:00 to $17: 00$, there is a local electricity valley time. At this time, DE is still running at higher power, while the power of FC is reduced. After the battery is discharged at the peak of power consumption in the morning, it is charged at this stage to store the energy for the late peak. After 18:00, electricity consumption begins to rise again and reaches the 


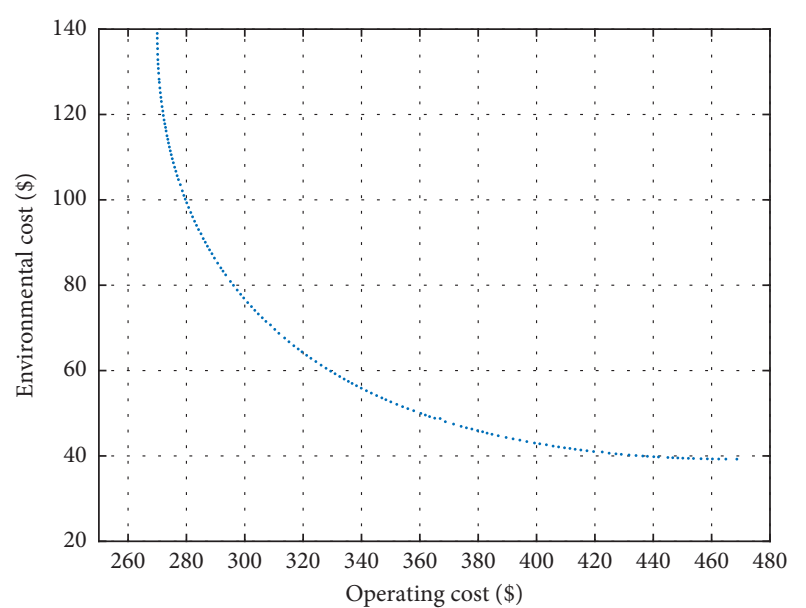

Figure 6: Pareto optimal front obtained by MOBBO-AM algorithm.

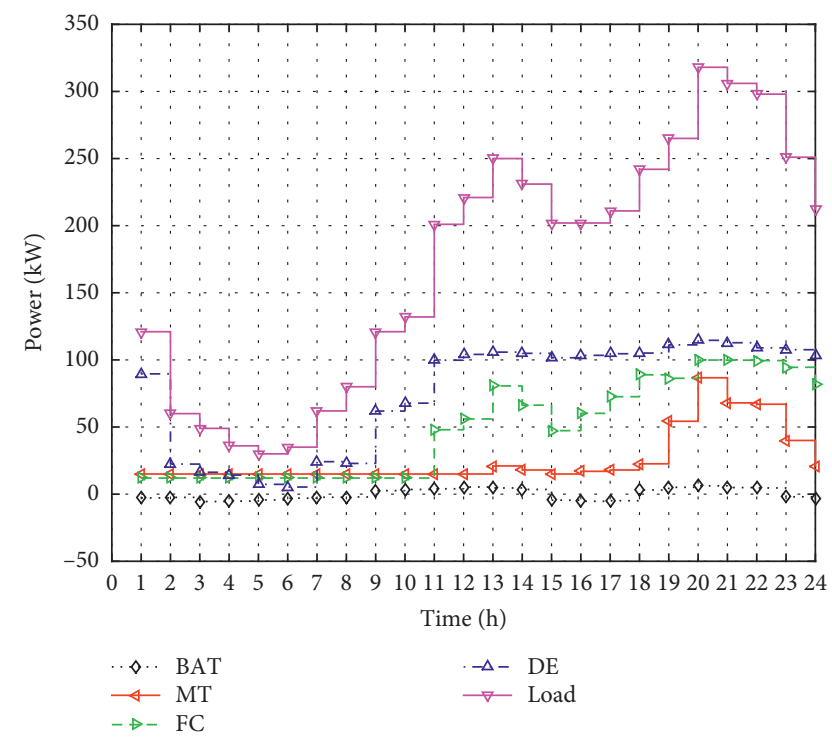

Figure 7: Results of the economic optimal scheduling.

TABle 2: Cost of different objectives.

\begin{tabular}{lcc}
\hline Objective function & $\begin{array}{c}\text { Operating } \\
\text { cost } / \$\end{array}$ & $\begin{array}{c}\text { Environmental } \\
\text { cost } / \$\end{array}$ \\
\hline $\begin{array}{l}\text { Minimum operating cost } \\
\text { Minimum environmental } \\
\text { cost }\end{array}$ & 269.9 & 138.9 \\
$\begin{array}{l}\text { Minimum comprehensive } \\
\text { cost }\end{array}$ & 468.5 & 39.25 \\
\hline
\end{tabular}

peak time in the whole day at 20:00, when there is no light and no PV output, and WT remains continuously powered. At this time, FC and DE are basically close to power operation in full load. The battery begins to discharge, while MT also operates with a higher power to meet the demand for electricity load. After 23:00, the electricity consumption decreases, the remaining capacity of the battery reaches the lower limit, and the battery starts to be charged.

The operating scenario in Figure 7 is the case of minimized operating cost. It can be seen that when cooperating with the PV and WT output, DE with the lowest operating cost is preferred, followed by FC, and finally MT is used as the compensation power demand in peak period. This scenario makes extensive use of DE, so its environmental cost is higher.

As can be seen from Figure 8, this operating scenario is to minimize the environmental cost. Unlike Figure 6, when FC can meet the power demand, MT and DE operate at lower power. And when FC power is insufficient, MT is called upon first to supplement power generation, and DE participates in power generation only during the peak period of the whole day to ensure the electricity demand. In this scenario, the DE with the highest environmental cost is used with the least possibility, but FC and MT are kept running at higher power, so the operating cost is higher.

The operating scenario in Figure 9 is the case of optimal comprehensive cost, that is, the compromise solution in Pareto front. It can be seen that when the demand for electricity is low, the output of using FC to cooperate with PV and WT is the main output, while when the demand for electricity is higher, the output of FC is the main output, followed by DE, and MT is used as a deficiency supplement. This scenario takes into account the low operating cost of DE and the low environmental cost of FC and MT, which is the comprehensive optimal scenario combining the operating cost and environmental cost.

It can be seen from Table 2 that, when the operating cost is minimum, the power generation cost of microgrid increases because of increasing the input cost of diesel generator sets with high pollutant emissions, which leads to the increase of environmental cost. When the environmental cost is minimum, the cost of pollutant treatment leads to the relative increase in operating cost. When the comprehensive cost objective is minimum, its environment cost and operating cost are in a limited range, and then it is necessary to balance various factors such as the distribution of wind and light resources, fuel prices, and pollutant treatment costs and choose a reasonable dispatching scheme when optimizing scheduling. It is likely to obtain the optimal solution with lower comprehensive operation cost or equivalent comprehensive cost but less environmental cost by selecting the compromise solution on Pareto frontier, which not only reduces the environmental impact but also maximizes the economy.

Then, in order to further verify the performance of the proposed MOBBO-AM algorithm, it is compared with two typical constrained multiobjective optimization algorithms. And for the purpose of proving the effectiveness and advancement of the proposed algorithm, we first compare the algorithm on a standard test set, and its performance is compared with that of NSGA-II [24] and NSDE [28] on the representative standard test problems [31] of TNK, SRN, CONSTR, and OSY. The convergence and distribution performance of the algorithms are tested using two evaluation criteria [32]: generational distance metric (GD) and 


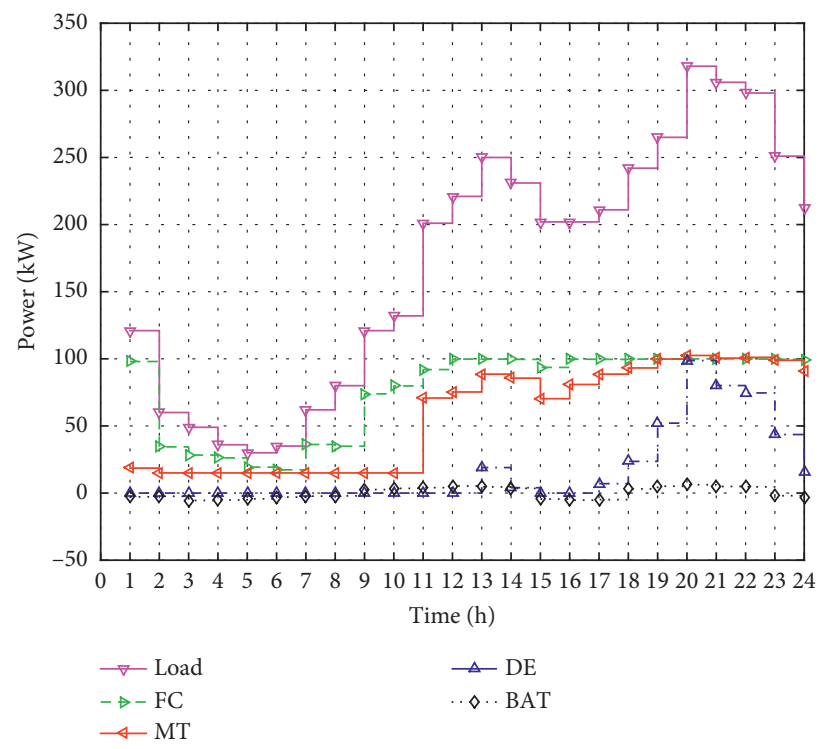

FIgURE 8: Results of the environmental optimal scheduling.

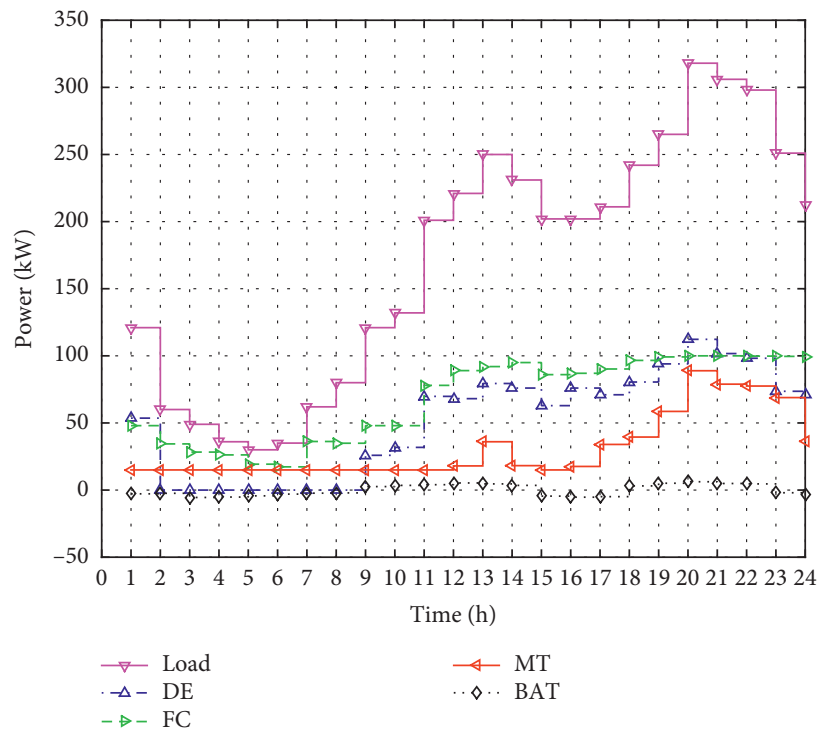

Figure 9: Results of the comprehensive optimal scheduling.

TABLe 3: Statistical results of GD indicator.

\begin{tabular}{lccc}
\hline Test function & MOBBO-AM & NSGA-II & NSDE \\
\multirow{2}{*}{ TNK } & $9.62 E-05$ & $7.80 E-04$ & $7.82 E-04$ \\
& $(2.06 E-05)$ & $(1.11 E-04)$ & $(1.18 E-04)$ \\
\hline \multirow{2}{*}{ SRN } & $3.77 E-04$ & $2.29 E-03$ & $1.31 E-03$ \\
& $(9.21 E-05)$ & $(8.07 E-04)$ & $(5.53 E-04)$ \\
\multirow{2}{*}{ CONSTR } & $6.59 E-05$ & $7.13 E-04$ & $3.97 E-04$ \\
& $(1.11 E-05)$ & $(1.02 E-04)$ & $(1.36 E-04)$ \\
\multirow{2}{*}{ OSY } & $6.03 E-04$ & $1.28 E-02$ & $7.11 E-03$ \\
& $(8.91 E-05)$ & $(2.69 E-03)$ & $(3.93 E-04)$ \\
\hline
\end{tabular}

the space metric (SP). To ensure the fairness of the comparison, each algorithm runs independently for 30 times. Table 3 shows the experimental statistical results of the three algorithms on the GD index of 4 standard test functions, including the average value (outside the parenthesis) and standard deviation (inside the parenthesis), which are used 


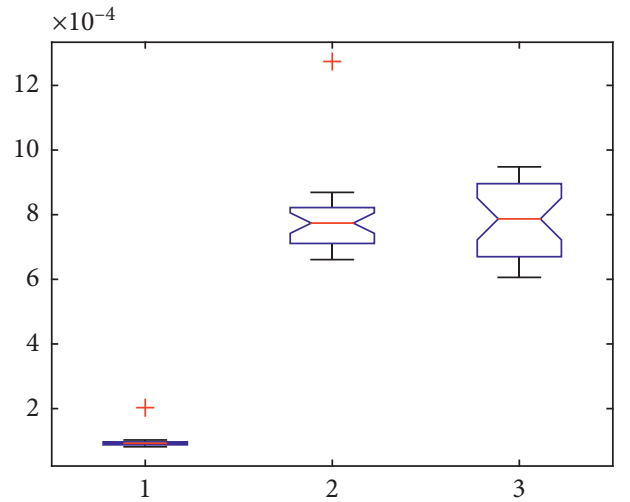

(a)

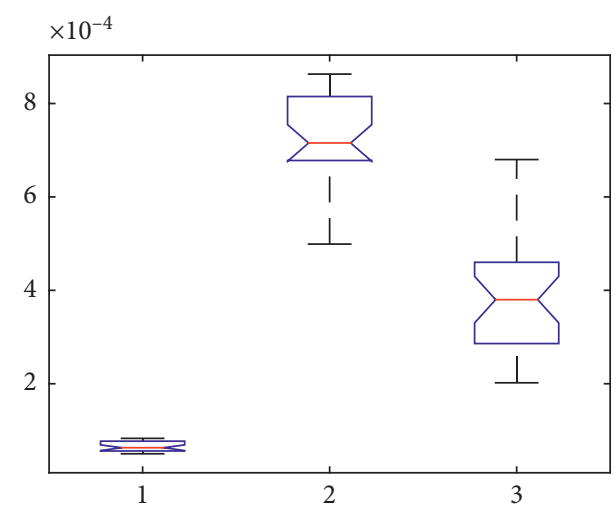

(c)

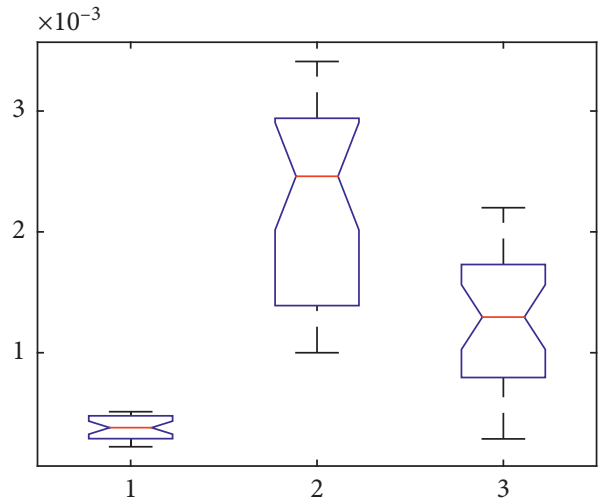

(b)

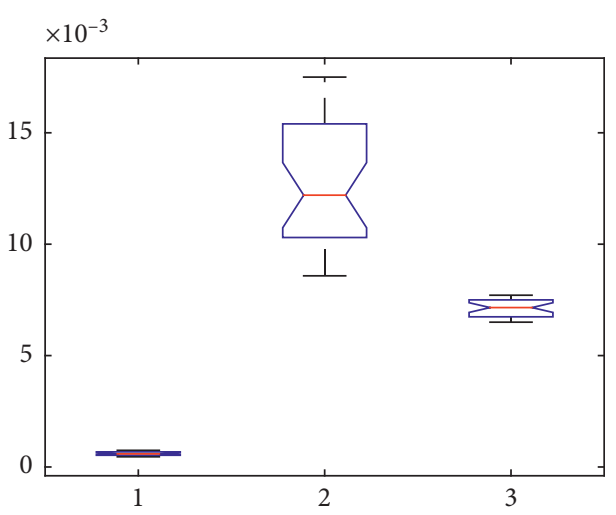

(d)

Figure 10: Statistical boxplots of GD indicator. (a) TNK, (b) SRN, (c) CONSTR, and (d) OSY.

to quantitatively measure the convergence performance of the solution set. The statistical box plots of MOBBO-AM and the other two algorithms are shown in Figure 10, where MOBBO-AM, NSGA-II, and NSDE are represented by numbers 1 to 3 , respectively.

It can be seen from Table 3 and Figure 10 that, for the 4 test functions, MOBBO-AM can not only obtain the minimum average GD value compared to NSGA-II and NSDE but also obtain the minimum GD value which is significantly smaller than that obtained by other algorithms. This shows that the results obtained by MOBBO-AM are closer to the ideal Pareto frontier and has an obvious advantage in convergence. Meanwhile, Figure 10 also shows that the statistical data obtained by MOBBO-AM also has fewer outliers than the other algorithms, which indicates that MOBBO-AM has the best convergence stability among the 3 algorithms.

For the distribution of the solution sets obtained by the three algorithms, the experimental statistical results of SP index are given in Table 4, and the statistical boxplots are shown in Figure 11.

From Table 4 and Figure 11, it is not difficult to see that MOBBO-AM obtains the smallest SP value and the least outliers in the statistical data for the 4 test functions, which indicates that MOBBO-AM has more advantages than the other two algorithms in terms of the uniformity of
TABLE 4: Statistical results of SP indicator.

\begin{tabular}{lccc}
\hline Test function & MOBBO-AM & NSGA-II & NSDE \\
\hline \multirow{2}{*}{ TNK } & $7.18 E-03$ & $1.92 E-02$ & $1.86 E-02$ \\
& $(1.39 E-03)$ & $(7.25 E-03)$ & $(4.91 E-03)$ \\
\hline \multirow{2}{*}{ SRN } & $5.80 E-03$ & $3.65 E-02$ & $2.61 E-02$ \\
& $(2.93 E-04)$ & $(1.31 E-02)$ & $(6.68 E-03)$ \\
\hline \multirow{2}{*}{ CONSTR } & $4.24 E-02$ & $8.85 E-02$ & $7.61 E-02$ \\
& $(3.94 E-03)$ & $(1.51 E-02)$ & $(7.37 E-03)$ \\
\hline \multirow{2}{*}{ OSY } & $1.11 E-01$ & $7.67 E-01$ & $6.89 E-01$ \\
& $(2.00 E-02)$ & $(2.01 E-01)$ & $(6.47 E-02)$ \\
\hline
\end{tabular}

distribution and can make the distribution of approximate Pareto optimal feasible solution set more uniform.

Summarizing the above experiments on the standard test functions, MOBBO-AM outperforms the other 2 algorithms in terms of both convergence and uniformity of distribution. Next, we use the algorithm for solving the optimized scheduling model for microgrids. The MOBBO-AM algorithm proposed in this paper is shown in Figure 6 and the simulation results of the NSGA-II and NSDE algorithms involved in the comparison are shown in Figures 12 and 13.

As can be seen from the figure, the optimization result of the proposed algorithm is that the solution sets are very evenly distributed on Pareto optimal frontier, while the Pareto optimal frontier of microgrid optimal scheduling 


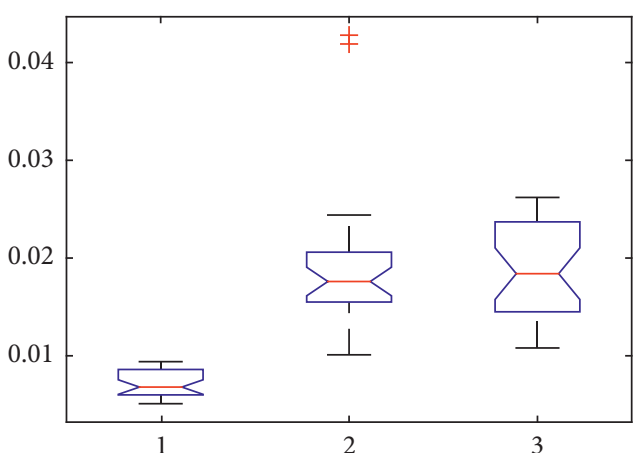

(a)

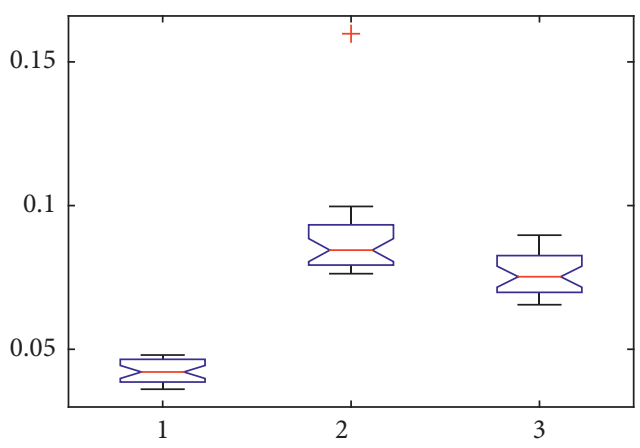

(c)

FIgURE 11: Statistical boxplots of SP indicator.

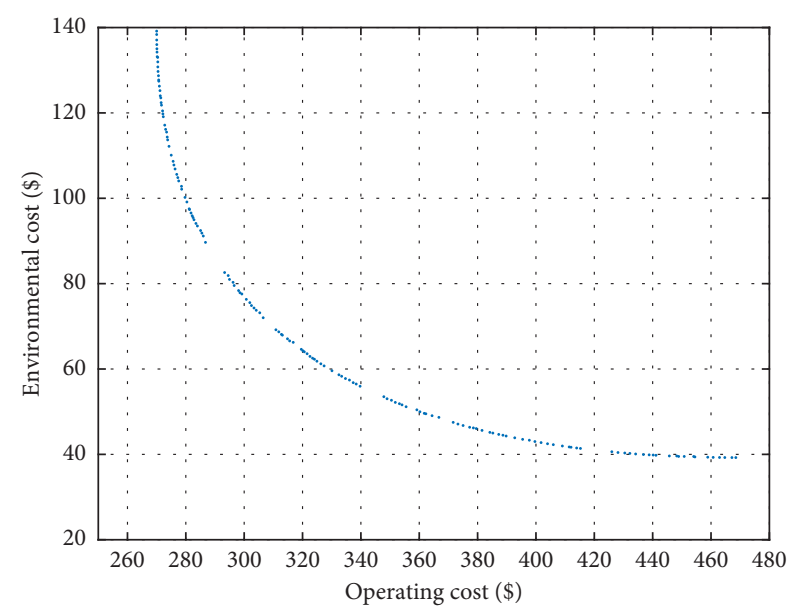

FIGURE 12: Pareto optimal front obtained by NSGA-II.

based on NSGA-II and NSDE makes the assemblage and discontinuity of solution set in different degrees, and the distribution uniformity is obviously inferior to the proposed MOBBO-AM. Therefore, compared with the comparative algorithm, the proposed algorithm can more effectively and comprehensively reflect the contradiction between the environmental cost and the operation cost of microgrid. As the basis for guiding the multiobjective optimization operation and research of microgrid, the optimization quality of the Pareto optimal solution set directly determines the selection of operation scheme. Hence, obtaining the Pareto optimal

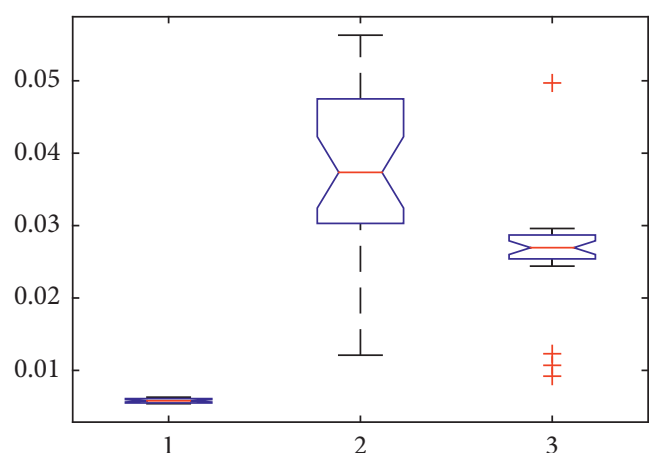

(b)

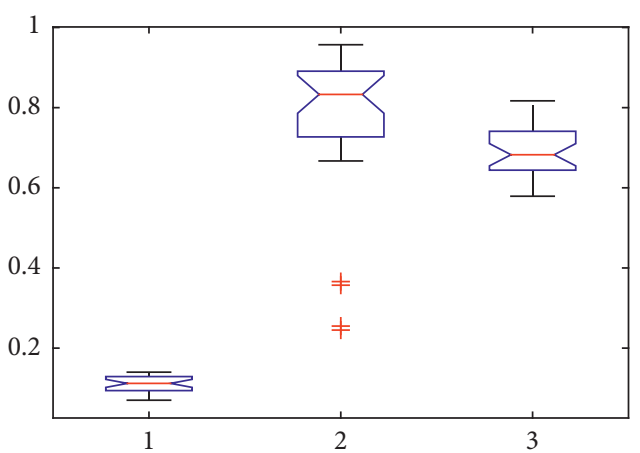

(d)

a) TNK, (b) SRN, (c) CONSTR, and (d) OSY.

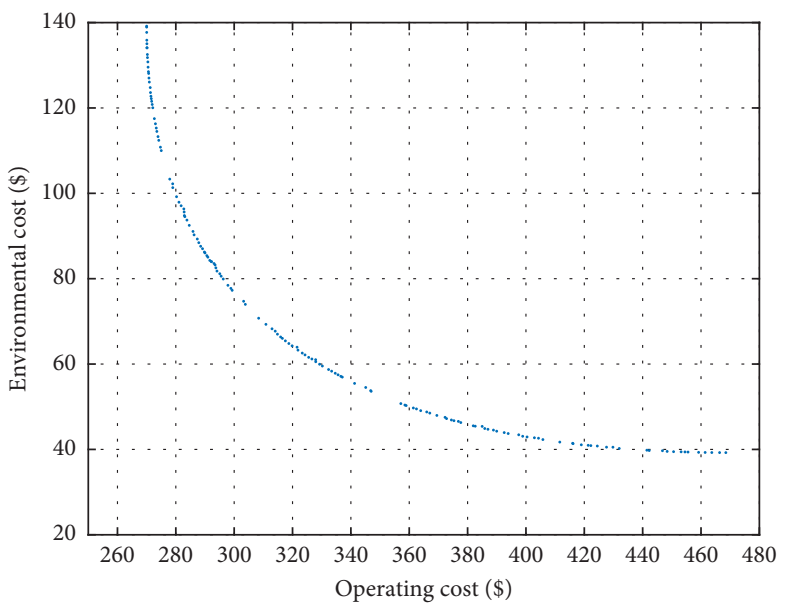

FIGURE 13: Pareto optimal front obtained by NSDE.

frontier with excellent uniformity and convergence performance can provide a more complete selection for the operation scheme, without missing the better compromise solution which is more in line with the actual demand owing to the discontinuity of the solution set.

\section{Conclusion}

In this paper, the dynamic optimal scheduling problem of microgrid in island operation is studied, and a multiobjective optimal scheduling model considering operating cost and environmental treatment cost is established. Taking 
a specific microgrid system including wind, light, storage battery, microgas turbine, fuel cell, diesel engine, and load as an example, constraint multiobjectives and biogeographybased optimization algorithm are organically integrated, and the corresponding constraint handling mechanism and the determination method of habitat suitability index with adaptive migration strategy are effectively improved. The simulation results show that the optimal scheduling algorithm based on multiobjective biogeography-based optimization with adaptive migration proposed in this paper is significantly superior and it can simply and quickly achieve global constrained multiobjective optimization to obtain accurate and complete Pareto frontier. According to biobjective operation results of operating cost and environmental cost, it can effectively better guide the optimal operation of microgrid according to the actual situation and play a certain guiding role in ensuring the islanded operation of microgrid and uninterrupted power supply for sensitive loads in microgrid, which can provide a reference for the economic and environmental protection scheduling of energy conservation and emission reduction.

\section{Data Availability}

The data used to support the findings of this study are available from the corresponding author upon request.

\section{Conflicts of Interest}

The authors declare that there are no conflicts of interest.

\section{Acknowledgments}

This work was supported in part by the Natural Science Foundation Program of Liaoning Province of China under Grants 20170520389 and 2019-ZD-0289.

\section{References}

[1] R. B. Palma, C. Benavides, F. Lanas et al., "A microgrid energy management system based on the rolling horizon strategy," IEEE Transactions on Smart Grid, vol. 4, no. 2, pp. 996-1006, 2013.

[2] M. H. Amrollahi and S. M. T. Bathaee, "Techno-economic optimization of hybrid photovoltaic/wind generation together with energy storage system in a stand-alone micro-grid subjected to demand response," Applied Energy, vol. 202, pp. 66-77, 2017.

[3] Y. X. Ma, W. Y. Wu, L. H. Fang, and Y. Sun, "Optimal sizing of hybrid solar-wind distributed generation in an island micro grid using improved bacterial foraging algorithm," Proceedings of the CSEE, vol. 31, no. 25, pp. 17-25, 2011.

[4] Y. Liang, F. Liu, C. Wang et al., "Distributed demand-side energy management scheme in residential smart grids: an ordinal state-based potential game approach," Applied Energy, vol. 206, pp. 991-1008, 2017.

[5] A. G. Mei and N. D. Hatziargyriou, "Centralized control for optimizing microgrids operation," IEEE Transactions on Energy Conversion, vol. 23, no. 1, pp. 241-248, 2008.

[6] E. Mashhour and S. M. Moghaddas-Tafreshi, "Integration of distributed energy resources into low voltage grid: a market- based multiperiod optimization model," Electric Power Systems Research, vol. 80, no. 4, pp. 473-480, 2010.

[7] C. Chen, S. Duan, T. Cai, B. Liu, and G. Hu, "Optimal allocation and economic analysis of energy storage system in microgrids," IEEE Transactions on Power Electronics, vol. 26, no. 10, pp. 2762-2773, 2011.

[8] A. F. Mohaed and N. H. Koivo, "System modelling and online optimal management of microgrid using mesh adaptive direct search," International Journal of Electrical Power \& Energy Systems, vol. 32, no. 5, pp. 398-407, 2010.

[9] C. D. Korkas, S. Baldi, I. Michailidis, E. B. Kosmatopoulos et al., "Intelligent energy and thermal comfort management in grid-connected microgrids with heterogeneous occupancy schedule," Applied Energy, vol. 149, no. 1, pp. 194-203, 2015.

[10] M. Marzband, H. Alavi, S. S. Ghazimirsaeid, H. Uppal et al., "Optimal energy management system based on stochastic approach for a home microgrid with integrated responsive load demand and energy storage," Sustainable Cities and Society, vol. 28, no. 9, pp. 256-264, 2017.

[11] M. Fernando, F. Shokridehaki, M. Marzband, R. Godina et al., "A two stage hierarchical control approach for the optimal energy management in commercial building microgrids based on local wind power and PEVs," Sustainable Cities and Society, vol. 41, no. 5, pp. 332-340, 2018.

[12] K. D. Pouresmaeil, B. Simone, and K. B. Elias, "Grid-connected microgrids: demand management via distributed control and human-in-the-loop optimization," Advances in Renewable Energies and Power Technologies, vol. 2, pp. 315344, 2018.

[13] N. Prashant and S. D. Joseph, "Techno-economic optimization and environmental life cycle assessment (LCA) of microgrids located in the US using genetic algorithm," Energy Conversion and Management, vol. 181, no. 1, pp. 272-291, 2019.

[14] J. P. T. Keerachat and T. Panida, "Mitigating microgrid voltage fluctuation using battery energy storage system with improved particle swarm optimization," Energy Reports, vol. 6, no. 2, pp. 724-730, 2020.

[15] R. Kallol, M. K. Kamal, M. C. Atis, and P. N. Sankar, "Analysis of energy management in micro grid-a hybrid BFOA and ANN approach," Renewable and Sustainable Energy Reviews, vol. 82, no. 3, pp. 4296-4308, 2018.

[16] B. C. Li, Y. J. Zhang, and P. Li, "Multi-objective optimization model of micro-grid operation considering cost, pollution discharge and risk," Proceedings of the CSEE, vol. 34, no. 19, pp. 3073-3079, 2014.

[17] J. Chen, X. Yang, L. Zhu, and M. Zhang, "Microgrid multiobjective economic dispatch optimization," Proceedings of the CSEE, vol. 33, no. 19, pp. 57-66, 2013.

[18] J. D. Chen, W. Q. Gong, L. M. Zhang et al., "Multi-objective optimal dispatch in wind power integrated system incorporating energy-environmental efficiency," Proceedings of the CSEE, vol. 31, no. 13, pp. 10-17, 2011.

[19] L. Zhao, G. Ju, and J. H. Lu, "An improved genetic algorithm in multi-objective optimization and its application," Proceedings of the CSEE, vol. 28, no. 2, pp. 96-102, 2008.

[20] A. Faisal, Mohamed, and K. N. Heikki, "Online management of microgrid with battery storage using multi-objective optimization," in Proceedings of the 2007 International Conference on Power Engineering, Energy and Electrical Drives, pp. 231-236, Setubal, Portugal, April 2007.

[21] P. Li, N. W. Xu, Y. Z. Zhou, and R. Li, "Optimal operation of microgrid based on improved gravitational search algorithm," Proceedings of the CSEE, vol. 34, no. 19, pp. 3073-3079, 2014. 
[22] H. Z. Zheng, Q. Ai, H. W. Xu et al., "A multi-objective dispatch optimization strategy for economic operation of smart grids," Power System Technology, vol. 34, no. 2, pp. 7-13, 2010.

[23] S. Mohammadi, B. Mozafari, S. Solimani et al., "An adaptive modified firefly optimisation algorithm based on hong's point estimate method to optimal operation management in a microgrid with consideration of uncertainties," Energy, vol. 51, no. 1, pp. 339-348, 2013.

[24] J. Niknam, D. D. Xu, F. J. Liu, and C. Li, "Multi-objective optimal operation of microgrid considering dynamic loads," Proceedings of the CSEE, vol. 36, no. 12, pp. 3325-3333, 2016.

[25] A. A. Moghaddam, A. Seifi, and T. Niknam, "Multi-operation management of a typical micro-grids using particle swarm optimization: a comparative study: a comparative study," Renewable and Sustainable Energy Reviews, vol. 16, no. 2, pp. 1268-1281, 2012.

[26] H. Borhanazad, S. Mekhilef, V. Gounder Ganapathy et al., "Optimization of micro-grid system using MOPSO," Renewable Energy, vol. 71, pp. 295-306, 2014.

[27] C.-S. Modiri-Delshad, G. Kyriakarakos, K. G. Arvanitis et al., "A multi-agent decentralized energy management system based on distributed intelligence for the design and control of autonomous polygeneration microgrids," Energy Conversion and Management, vol. 103, pp. 166-179, 2015.

[28] H. C. Papadakis and J. H. Sun, "Multi-objective optimization power dispatch based on non-dominated sorting differential evolution," Proceedings of the CSEE, vol. 29, no. 34, pp. 71-76, 2009.

[29] F. A. Mohamed and H. N. Koivo, "Multiobjective optimization using modified game theory for online management of microgrid," European Transactions on Electrical Power, vol. 21, no. 1, pp. 839-854, 2011.

[30] D. Simon, "Biogeography-based optimization," IEEE Transactions on Evolutionary Computation, vol. 12, no. 6, pp. 702-713, 2008.

[31] A. Angantyr, J. Andersson, and J. Aidanpaa, "Constrained optimization based on a multi-objective evolutionary algorithm," in Proceedings of the 2003 IEEE Congress on Evolutionary Computation, vol. 1, pp. 1560-1567, Canberra, Australia, December 2003.

[32] L. Tang and X. Wang, "A hybrid multiobjective evolutionary algorithm for multiobjective optimization problems," IEEE Transactions on Evolutionary Computation, vol. 17, no. 1, pp. 20-45, 2013. 